\title{
MAPEAMENTO EPIDEMIOLÓGICO DAS HEPATITES HOSPITALARES
}

\section{Epidemiological mapping of hospital hepatites \\ Mapeo epidemiológico de las hepatitis hospitalarias}

\author{
Lígia Maria Cabedo Rodrigues \\ Universidade Federal do Piauí - UFPI - Teresina (PI) - Brasil \\ Érida Zoé Lustosa Furtado iD \\ Hospital Universitário da Universidade Federal do Piauí - UFPI - Teresina (PI) - Brasil
}

Anne Karoline Nunes de Oliveira

Universidade Federal do Piauí - UFPI - Teresina (PI) - Brasil

Jaqueline da Cunha Morais (iD

Universidade Federal do Piauí - UFPI - Teresina (PI) - Brasil

Maria Tainara dos Santos Resende (iD

Universidade Federal do Piauí - UFPI - Teresina (PI) - Brasil

Vanessa Rodrigues da Silva

Universidade Federal do Piauí - UFPI - Parnaíba (PI) - Brasil

\section{RESUMO}

Objetivo: Analisar os aspectos sociodemográficos e epidemiológicos de pacientes com hepatite $\mathrm{B}$ e/ou $\mathrm{C}$ atendidos em um hospital universitário do Nordeste brasileiro. Métodos: Estudo transversal, descritivo e analítico, realizado no Hospital Universitário do Piauí com 98 pacientes diagnosticados com hepatite B e/ou C de julho/2014 a dezembro/2017. Os dados foram coletados a partir das fichas de investigação de hepatites virais e analisados através do SPSS. Resultados: A maioria dos participantes tinha mais de 60 anos $(48,0 \%, n=47)$, era do sexo masculino $(67,3 \%, n=66)$, de cor parda $(77,6 \%, n=76)$, com ensino fundamental completo ou menos $(58,1 \%, n=57)$, procedente da zona urbana $(88,8 \%, n=87)$ e aposentado $(28,6 \%$, $n=28)$. Verificou-se que $58,2 \%(n=57)$ não possuía vacinação contra hepatite $B$ e, entre os portadores do vírus $C$, prevaleceram os genótipos 1 e 3 . A hepatite crônica se fez presente em $75,5 \%(n=74)$ dos casos e como fontes mais frequentes de infecção predominaram a via transfusional e sexual e o uso de drogas. Identificou-se associação estatística significativa para o vírus $\mathrm{B}$ entre: pacientes com idade de 40 a 59 anos, procedentes de outros municípios, de zona urbana e transmissão sexual; e, para o vírus C, entre: idosos, procedentes de Teresina, de zona urbana, forma crônica da doença e transmissão transfusional. Conclusão: Constatou-se uma maior prevalência das hepatites virais hospitalares em pacientes idosos, do sexo masculino e com baixa escolaridade, que já se apresentava na forma crônica da doença, sendo o vírus $\mathrm{C}$ o mais frequente.

Descritores: Hepatite B; Hepatite C; Epidemiologia; Notificação de Doenças.

\section{ABSTRACT}

Objective: To analyze the sociodemographic and epidemiological aspects of patients with hepatitis $B$ and/or $C$ treated at a university hospital in Northeastern Brazil. Methods: This descriptive and analytical cross-sectional study was conducted at the Piaui University Hospital with 98 patients diagnosed with hepatitis B and/or C between July 2014 to December 2017. Data were collected from viral hepatitis investigation sheets and analyzed using the SPSS. Results: Most participants were over 60 years old (48.0\%, $n=47)$, male (67.3\%, $n=66)$, pardos (Mixed-race Brazilians) $(77.6 \%, n=76)$, had completed primary education or less $(58.1 \%, n=57)$, and were originally from urban areas $(88.8 \%, n=87)$ and retired $(28.6 \%, n=28)$. In all, $58.2 \%(n=57)$ did not get hepatitis $B$ vaccination and genotypes 1 and 3 were prevalent among carriers of virus $C$. Chronic hepatitis was present in $75.5 \%$ $(n=74)$ of the cases and the most frequent sources of infection were transfusion, sex and drug use. Virus $B$ was significantly associated with: patients aged 40 to 59 years, other municipalities, urban area and sexual transmission. Virus $C$ was significantly associated with: older adults, the city of Teresina, urban area, chronic form of the disease and transfusion. Conclusion: There was 
a higher prevalence of hospital viral hepatitis in older patients, male patients, patients with low levels of education, and patients who already had the chronic form of the disease, the most common being virus $C$.

Descriptors: Hepatitis B; Hepatitis C; Epidemiology; Disease Notification.

\section{RESUMEN}

Objetivo: Analizar los aspectos sociodemográficos y epidemiológicos de pacientes con la hepatitis $B$ y/o $C$ asistidos en un hospital del Noreste brasileño. Métodos: Estudio transversal, descriptivo y analítico realizado en el Hospital Universitario de Piauí con 98 pacientes con el diagnostico de hepatitis B y/o C entre julio/2014 y diciembre/2017. Se recogieron los datos de las fichas de investigación de hepatitis virales y se les han analizados por el SPSS. Resultados: La mayoría de los participantes tenía más de 60 años $(48,0 \%, n=47)$, era del sexo masculino $(67,3 \%, n=66)$, de color pardo $(77,6 \%, n=76)$, con educación primaria completa o menos $(58,1 \%, n=57)$, de la zona urbana $(88,8 \%, n=87)$ y jubilado $(28,6 \%, n=28)$. Se verificó que el $58,2 \%(n=57)$ no se había vacunado contra la hepatitis $B$ y entre los portadores del virus $C$ han prevalecido los genotipos 1 y 3 . La hepatitis crónica estuvo presente en el $75,5 \%(n=74)$ de los casos y la vía transfusional, y la sexual y el uso de drogas han sido las fuentes más frecuentes de infección. Se identificó una asociación estadística significante para el virus $B$ entre los pacientes de edad entre 40 y 59 años que eran de otros municipios, de la zona urbana y por transmisión sexual; y para el virus $C$ entre mayores que venían de Teresina, de la zona urbana, con la forma crónica de la enfermedad y transmisión transfusional. Conclusión: Se ha constatado una mayor prevalencia de las hepatitis virales hospitalarias en pacientes mayores, del sexo masculino y de baja escolaridad que ya tenía la forma crónica de la enfermedad y el virus $C$ como el más frecuente.

Descriptores: Hepatitis B; Hepatitis C; Epidemiología; Notificación de Enfermedades.

\section{INTRODUÇÃO}

As hepatites B e C são infecções que impactam a saúde pública mundial. Transmitidas de diferentes formas, possuem características clínicas, laboratoriais e epidemiológicas específicas, com prevalência e magnitude que variam de região para região. $\mathrm{O}$ impacto dessas infecções está diretamente relacionado ao aumento do número de pessoas com hepatite $\mathrm{B}$ e $\mathrm{C}$ e com a possibilidade de cronificação da doença, com consequente perda da qualidade de vida do paciente ${ }^{(1,2)}$.

As hepatites virais apresentam altas taxas de morbimortalidade, principalmente relacionadas à evolução da doença, que pode ir de formas assintomáticas, agudas, até a cronificação. São responsáveis por cerca de 1,4 milhão de óbitos anualmente no mundo, decorrentes, principalmente, das complicações da forma descompensada crônica ou do hepatocarcinoma ${ }^{(3)}$. Isto se deve, muitas vezes, a fatores como: heterogeneidade socioeconômica, distribuição irregular dos serviços de saúde e incorporação desigual de tecnologias que viabilizem diagnósticos e tratamentos precoces e adequados ${ }^{(4)}$.

No Brasil e no mundo, o comportamento das hepatites virais tem apresentado grandes mudanças nos últimos anos. A melhoria nas condições de higiene e de saneamento básico das populações, a vacinação contra a hepatite $B$ e as novas técnicas moleculares de diagnóstico da hepatite $C$ constituem fatores importantes, que se vinculam às transformações no perfil dessas doenças ${ }^{(5)}$.

A vigilância epidemiológica dessas doenças no país é baseada na notificação compulsória dos casos suspeitos desde 1996. Entre 1999 a 2017, foram notificados, conforme o Sistema de Informação de Agravos de Notificação (SINAN), 587.821 casos confirmados de hepatites virais no Brasil, dos quais $218.257(37,1 \%)$ foram de hepatite B e 200.839 (34,2\%) correspondentes à hepatite C. De 2000 a 2016, foram identificados, no Brasil, pelo Sistema de Informação de Mortalidade, 66.196 óbitos associados às hepatites virais, sendo que $21,4 \%$ correspondiam à hepatite $\mathrm{B}$ e $75,8 \%$, à hepatite $\mathrm{C}^{(6)}$.

Seguindo os eixos operacionais da Política Nacional de Promoção da Saúde ${ }^{(7)}$, a vigilância atua no controle das hepatites virais através do monitoramento do comportamento das mesmas e seus fatores condicionantes e determinantes, com a finalidade de recomendar e adotar medidas de prevenção e controle, e avaliar o seu impacto.

A epidemiologia da hepatite $B$ não é homogênea no cenário nacional e as áreas em que há dificuldade de acesso aos serviços de saúde são desproporcionalmente afetadas. Trata-se de doença causada por um vírus resistente, que chega a sobreviver sete dias no ambiente externo, em condições normais e com risco de, em casos de contato com sangue, levar a infecção para $5,0 \%$ a $40,0 \%$ das pessoas não vacinadas. Cerca de $50,0 \%$ dos casos de hepatite fulminante estão relacionados à infecção por hepatite $\mathrm{B}^{(8)}$. 
A hepatite $C$, por sua vez, representa impacto significativo para a saúde pública em decorrência da progressão da doença e de suas complicações, pois apresenta evolução silenciosa, diagnóstico tardio e, nas fases avançadas, o paciente pode evoluir para óbito. Estima-se que, no mundo, a hepatite $\mathrm{C}$ seja responsável por aproximadamente 700.000 óbitos anuais ${ }^{(9)}$.

Diante da relevância e do impacto dessas doenças, este estudo objetivou analisar os aspectos sociodemográficos e epidemiológicos de pacientes com hepatite B e/ou C atendidos em um hospital universitário no Nordeste brasileiro.

\section{MÉTODOS}

Trata-se de estudo com delineamento transversal, retrospectivo, descritivo e analítico, desenvolvido no Hospital Universitário da Universidade Federal do Piauí (HU-UFPI) em 2018. O HU-UFPI localiza-se em Teresina, Piauí, Brasil, atua na prestação de serviços de alta e média complexidade para a população a partir de 18 anos de idade e oferta atendimento ambulatorial de diversas especialidades médicas, como a gastroenterologia, que atende e acompanha regularmente pacientes portadores de hepatites virais, o que tornou o HU-UFPI referência para tratamento dessa doença. Casos crônicos de hepatites que requerem acompanhamento clínico através de internação são também comumente hospitalizados nessa instituição.

O encaminhamento de pacientes para serviços ambulatoriais é regulado pela Secretaria Municipal de Saúde, por meio de agendamento pela central de marcação de consultas do SUS. A regulação de pacientes para a internação, por sua vez, é de responsabilidade da Central Estadual de Regulação e ocorre de acordo com a disponibilidade de vagas, que é informada diariamente à referida central.

Dentro do HU-UFPI, a Vigilância em Saúde, com equipe formada por médicos, enfermeiros e técnicos de enfermagem, é o setor responsável por notificar os casos suspeitos/confirmados de hepatites virais atendidos; acompanhar a realização de exames laboratoriais específicos e a liberação dos resultados; encaminhar as fichas de notificação ao serviço de epidemiologia municipal para posterior digitação no SINAN, como também arquivar uma via das notificações para controle e organização do setor.

Nesse contexto, a população deste estudo foi composta pelos pacientes diagnosticados com hepatite B e/ou C de julho de 2014 a dezembro de 2017. Os dados foram coletados a partir das fichas de investigação de hepatites virais do SINAN dos casos notificados, disponíveis no Setor de Vigilância em Saúde do HU-UFPI.

Os critérios para inclusão compreenderam: pacientes atendidos no ambulatório ou que estiveram internados no HU-UFPI, que foram notificados/investigados para hepatites virais nesse serviço e com resultados de exames sorológicos confirmatórios para hepatite B e/ou C.

Como instrumento de coleta de dados, utilizou-se formulário contendo características sociodemográficas e epidemiológicas dos pacientes notificados, elaborado a partir das informações da ficha de investigação de hepatites virais do SINAN.

Para a caracterização da população, realizou-se um estudo estatístico, descritivo e analítico da amostra através das frequências absolutas (n) e relativas (\%). Para verificar se as variáveis envolvidas eram independentes ou se existia alguma associação entre elas utilizou-se o teste qui-quadrado de independência $\left(X^{2}\right)$ e o teste exato de Fisher.

Os dados foram tabulados em planilha eletrônica Microsoft Office Excel e analisados no programa Statistical Package for the Social Sciences, versão 20.0. O nível de significância adotado foi de $\alpha=0,05$. Para apresentação dos resultados, utilizaram-se tabelas.

A pesquisa recebeu aprovação do Comitê de Ética em Pesquisa do HU-UFPI (Parecer $n .^{\circ}: 2.413 .579$ ), obedecendo aos princípios ético-legais norteadores de pesquisas envolvendo seres humanos, dispostos na Resolução 466/2012 do Conselho Nacional de Saúde.

\section{RESULTADOS}

A amostra englobou 98 casos de pacientes portadores de hepatite B/C. A maioria dos participantes tinha mais de 60 anos (48,0\%), era do sexo masculino $(67,3 \%)$, de cor/raça parda $(77,6 \%)$, com ensino fundamental completo ou menos $(58,1 \%)$. Quase $100,0 \%$ eram residentes no Piauí, procedentes da zona urbana $(88,8 \%)$, com destaque para a capital Teresina $(57,1 \%)$. A ocupação mais frequente foi aposentado $(28,6 \%)$ (Tabela I).

Quanto à distribuição dos antecedentes epidemiológicos, em 95,9\% dos pacientes havia suspeita de hepatites $B / C$, sendo que mais da metade não foram vacinados para hepatite $B(58,2 \%)$ e quase $96,0 \%$ não possuíam outras infecções sexualmente transmissíveis (IST) associadas. Em relação aos fatores de risco, observou-se que 
as exposições mais frequentes foram a medicamentos injetáveis $(78,6 \%)$, tratamento dentário $(72,4 \%)$, tratamento cirúrgico $(61,2 \%)$, três ou mais parceiros sexuais $(49,0 \%)$ e hemotransfusão $(26,5 \%)$ (Tabela II).

Tabela I - Distribuição dos dados sociodemográficos dos pacientes com hepatite B/C ( $n=98)$. Teresina, Piauí, 2018.

\begin{tabular}{|c|c|c|}
\hline Variáveis & $\mathbf{n}$ & $\%$ \\
\hline \multicolumn{3}{|l|}{ Idade } \\
\hline 20 a 39 anos & 12 & 12,2 \\
\hline 40 a 59 anos & 39 & 39,8 \\
\hline$>60$ anos & 47 & 48,0 \\
\hline \multicolumn{3}{|l|}{ Sexo } \\
\hline Masculino & 66 & 67,3 \\
\hline Feminino & 32 & 32,7 \\
\hline \multicolumn{3}{|l|}{ Cor/raça } \\
\hline Branca & 8 & 8,2 \\
\hline Preta & 13 & 13,3 \\
\hline Parda & 76 & 77,6 \\
\hline Amarela & 1 & 1,0 \\
\hline \multicolumn{3}{|l|}{ Escolaridade } \\
\hline Não alfabetizado & 17 & 17,3 \\
\hline Ensino fundamental incompleto/completo & 40 & 40,8 \\
\hline Ensino médio incompleto/completo & 32 & 32,7 \\
\hline Educação superior incompleta/completa & 9 & 9,2 \\
\hline \multicolumn{3}{|l|}{ Estado de Residência } \\
\hline Piauí & 95 & 97,0 \\
\hline Outros & 3 & 3,0 \\
\hline \multicolumn{3}{|l|}{ Procedência } \\
\hline Teresina & 56 & 57,1 \\
\hline Outros & 42 & 42,9 \\
\hline \multicolumn{3}{|l|}{ Zona } \\
\hline Rural & 11 & 11,2 \\
\hline Urbana & 87 & 88,8 \\
\hline \multicolumn{3}{|l|}{ Ocupação } \\
\hline Aposentado & 28 & 28,6 \\
\hline Lavrador & 16 & 16,3 \\
\hline Autônomo & 6 & 6,1 \\
\hline Desempregado & 6 & 6,1 \\
\hline Dona de casa & 5 & 5,1 \\
\hline Ignorado & 4 & 4,1 \\
\hline Vendedor & 3 & 3,1 \\
\hline Outros & 30 & 30,6 \\
\hline
\end{tabular}

Fonte: Fichas de investigação de hepatites virais, HU-UFPI, 2014-2017 
Tabela II - Distribuição dos antecedentes epidemiológicos dos pacientes com hepatite B/C ( $n=98)$. Teresina, Piauí, 2018.

\begin{tabular}{|c|c|c|}
\hline Variáveis & n & $\%$ \\
\hline \multicolumn{3}{|l|}{ Suspeita } \\
\hline Hepatite B/C & 94 & 95,9 \\
\hline Não especificada & 4 & 4,1 \\
\hline \multicolumn{3}{|l|}{ Tomou vacina para Hepatite B } \\
\hline Completa & 12 & 12,2 \\
\hline Incompleta & 10 & 10,2 \\
\hline Não vacinado & 57 & 58,2 \\
\hline Ignorado & 19 & 19,4 \\
\hline \multicolumn{3}{|l|}{ Agravos associados } \\
\hline Outras IST & 4 & 4,1 \\
\hline \multicolumn{3}{|l|}{ Paciente foi submetido } \\
\hline Medicamentos injetáveis & 77 & 78,6 \\
\hline Tratamento dentário & 71 & 72,4 \\
\hline Tratamento cirúrgico & 60 & 61,2 \\
\hline Três ou mais parceiros sexuais & 48 & 49,0 \\
\hline Transfusão de sangue/derivados & 26 & 26,5 \\
\hline Água/alimento contaminado & 23 & 23,5 \\
\hline Drogas inaláveis ou crack & 10 & 10,2 \\
\hline Drogas injetáveis & 10 & 10,2 \\
\hline Hemodiálise & 5 & 5,1 \\
\hline Tatuagem/piercing & 5 & 5,1 \\
\hline Transplante & 1 & 1,0 \\
\hline Outras & 5 & 5,1 \\
\hline
\end{tabular}

IST: infecções sexualmente transmissíveis. Fonte: Fichas de investigação de hepatites virais, HU-UFPI, 2014-2017

A Tabela III demonstra que, para o portador do vírus da hepatite C (HCV), foram identificados os genótipos 1,2 e 3 . A maior parte dos casos correspondeu ao genótipo $1(26,5 \%)$, seguido do genótipo $3(20,4 \%)$. Quanto à forma clínica das hepatites B e C, $75,5 \%$ dos casos identificados foram classificados como hepatite crônica/portador assintomático, sendo o vírus $C$ o mais frequente $(55,1 \%)$. Registra-se, ainda, a presença de um caso de paciente portador dos vírus B e C. Nos casos de pacientes em que foi possível definir o provável mecanismo da infecção $(n=30)$, as fontes mais frequentes foram a via transfusional $(10,2 \%)$, seguida da sexual $(8,2 \%)$ e do uso de drogas $(5,1 \%)$. 
Tabela III - Distribuição dos genótipos para o vírus da hepatite C (HCV), forma clínica, classificação etiológica e provável fonte de infecção dos pacientes com hepatite B/C ( $n=98)$. Teresina, Piauí, 2018.

\begin{tabular}{|c|c|c|}
\hline Variáveis & $\mathbf{n}$ & $\%$ \\
\hline \multicolumn{3}{|l|}{ Genótipo para HCV } \\
\hline Genótipo 1 & 26 & 26,5 \\
\hline Genótipo 2 & 3 & 3,1 \\
\hline Genótipo 3 & 20 & 20,4 \\
\hline Ignorado & 6 & 6,1 \\
\hline \multicolumn{3}{|l|}{ Forma clínica } \\
\hline Hepatite aguda & 24 & 24,5 \\
\hline Hepatite crônica/portador assintomático & 74 & 75,5 \\
\hline \multicolumn{3}{|l|}{ Classificação etiológica } \\
\hline Vírus B & 43 & 43,9 \\
\hline Vírus C & 54 & 55,1 \\
\hline Vírus B e C & 1 & 1,0 \\
\hline \multicolumn{3}{|l|}{ Provável fonte/mecanismo de Infecção } \\
\hline Transfusional & 10 & 10,2 \\
\hline Sexual & 8 & 8,2 \\
\hline Uso de drogas & 5 & 5,1 \\
\hline Tratamento cirúrgico & 4 & 4,1 \\
\hline Vertical & 3 & 3,1 \\
\hline Outros & 2 & 2,0 \\
\hline Ignorado & 66 & 67,3 \\
\hline
\end{tabular}

Fonte: Fichas de investigação de hepatites virais, HU-UFPI, 2014-2017

Os dados da Tabela IV, por sua vez, mostram que os pacientes com faixa etária de 40 a 59 anos tiveram maior chance de apresentar infecção pelo vírus $B(p<0,001)$, enquanto os idosos foram mais acometidos pelo vírus $C$ $(p<0,001)$. Observou-se, ainda, associação estatística significativa entre pacientes procedentes de outro município e resultado positivo para hepatite $B(p=0,044)$, enquanto a hepatite $C$ foi mais frequente entre os procedentes de Teresina $(p=0,011)$. A zona de residência urbana esteve estatisticamente associada às infecções pelos vírus $B$ e $C$.

Observa-se na Tabela $V$ que houve associação estatística significativa dos pacientes com vírus $C$ que apresentaram a forma crônica da doença $(p<0,002)$. Entre as prováveis fontes ou mecanismos de infecção registrados, observouse que a via sexual, a transmissão vertical e o tratamento cirúrgico apresentaram associação estatística significativa com a hepatite $B(p=0,008)$. Além disso, verificou-se associação estatística significativa entre as possíveis formas de transmissão por via transfusional, uso de drogas, tratamento cirúrgico e por via sexual com a infecção pelo vírus $C(p=0,015)$. 
Tabela IV - Associação do HBsAg e Anti-HCV com dados sociodemográficos dos pacientes com hepatite B/C (n=98). Teresina, Piauí, 2018.

\begin{tabular}{|c|c|c|c|c|c|c|}
\hline & & HBsAg & & & Anti-HCV & \\
\hline & $\begin{array}{l}\text { Positivo } \\
\text { n (\%) }\end{array}$ & $\begin{array}{c}\text { Negativo } \\
\text { n (\%) }\end{array}$ & p-valor & $\begin{array}{l}\text { Positivo } \\
\text { n (\%) }\end{array}$ & $\begin{array}{c}\text { Negativo } \\
\text { n (\%) }\end{array}$ & p-valor \\
\hline \multicolumn{7}{|l|}{ Idade } \\
\hline 20 a 39 anos & $11(25,0)$ & $1(2,7)$ & $<0,001^{a}$ & $1(1,8)$ & $7(24,1)$ & $<0,001^{a}$ \\
\hline 40 a 59 anos & $22(50,0)$ & $11(29,7)$ & & $17(30,9)$ & $15(51,7)$ & \\
\hline$>60$ anos & $11(25,0)$ & $25(67,6)$ & & $37(67,3)$ & $7(24,1)$ & \\
\hline \multicolumn{7}{|l|}{ Sexo } \\
\hline Masculino & $32(72,7)$ & $22(59,5)$ & $0,305^{a}$ & $35(63,6)$ & $22(75,9)$ & $0,371^{\mathrm{a}}$ \\
\hline Feminino & $12(27,3)$ & $15(40,5)$ & & $20(36,4)$ & $7(24,1)$ & \\
\hline \multicolumn{7}{|l|}{ Cor/raça } \\
\hline Branca & $3(6,8)$ & $2(5,4)$ & $0,956^{\mathrm{b}}$ & $5(9,1)$ & $2(6,9)$ & $0,601^{\mathrm{b}}$ \\
\hline Preta & $7(15,9)$ & $5(13,5)$ & & $6(10,9)$ & $4(13,8)$ & \\
\hline Parda & $33(75,0)$ & $30(81,1)$ & & $44(80,0)$ & $22(75,9)$ & \\
\hline Amarela & $1(2,3)$ & - & & - & $1(3,4)$ & \\
\hline \multicolumn{7}{|l|}{ Escolaridade } \\
\hline Não alfabetizado & $9(20,5)$ & $6(16,2)$ & $0,405^{b}$ & $8(14,5)$ & $8(27,6)$ & $0,344^{b}$ \\
\hline Ensino fundamental incompleto/completo & $18(40,9)$ & $14(37,8)$ & & $23(41,8)$ & $12(41,4)$ & \\
\hline Ensino médio incompleto/completo & $15(34,1)$ & $11(29,7)$ & & $17(30,9)$ & $8(27,6)$ & \\
\hline Ensino superior incompleto/completo & $2(4,5)$ & $6(16,2)$ & & $7(12,7)$ & $1(3,4)$ & \\
\hline \multicolumn{7}{|l|}{ Estado de residência } \\
\hline Piauí & $42(95,5)$ & $37(100,0)$ & $0,497^{b}$ & $53,2(96,4)$ & $29(100,0)$ & $0,542^{b}$ \\
\hline Outros & $2(4,5)$ & - & & $2(3,6)$ & - & \\
\hline \multicolumn{7}{|l|}{ Procedência } \\
\hline Teresina & $18(40,9)$ & $24(64,9)$ & $0,044^{\mathrm{a}}$ & $38(69,1)$ & $11(37,9)$ & $0,011^{a}$ \\
\hline Outros & $26(58,1)$ & $13(35,1)$ & & $17(30,9)$ & $18(62,1)$ & \\
\hline \multicolumn{7}{|l|}{ Zona de residência } \\
\hline Rural & $9(20,5)$ & $1(2,7)$ & $0,018^{a}$ & $2(3,6)$ & $5(17,2)$ & $0,045^{b}$ \\
\hline Urbana & $35(79,5)$ & $36(97,3)$ & & $53(96,4)$ & $24(82,8)$ & \\
\hline
\end{tabular}

a:Teste qui-quadrado; b:Teste exato de Fisher, Fonte: Fichas de investigação de hepatites virais, HU-UFPI, 2014-2017. HBsAg: antígeno de superfície do vírus da hepatite B. Indica infecção atual da hepatite B; Anti-HCV: detecta a hepatite C 
Tabela V - Associação do HBsAg e Anti-HCV com a forma clínica e provável fonte de infecção dos pacientes com hepatite B/C (n=98). Teresina, Piauí, 2018.

\begin{tabular}{|c|c|c|c|c|c|c|}
\hline & \multicolumn{3}{|c|}{ HBsAg } & \multicolumn{3}{|c|}{ Anti-HCV } \\
\hline & $\begin{array}{c}\text { Positivo } \\
\text { n (\%) }\end{array}$ & $\begin{array}{c}\text { Negativo } \\
\text { n (\%) }\end{array}$ & p-valor & $\begin{array}{c}\text { Positivo } \\
\text { n (\%) }\end{array}$ & $\begin{array}{c}\text { Negativo } \\
\text { n (\%) }\end{array}$ & p-valor \\
\hline \multicolumn{7}{|l|}{ Forma clínica } \\
\hline Hepatite aguda & $16(36,0)$ & $8(22,0)$ & $0,229^{a}$ & $8(15,0)$ & $14(48,0)$ & $0,002^{\mathrm{a}}$ \\
\hline Hepatite Crônica/ portador assintomático & $28(64,0)$ & $29(78,0)$ & & $47(85,0)$ & $15(52,0)$ & \\
\hline \multicolumn{7}{|l|}{ Provável fonte/ mecanismo de infecção } \\
\hline Sexual & $4(50,0)$ & $3(19,0)$ & $0,008^{b}$ & $4(16,0)$ & $3(75,0)$ & $0,015^{b}$ \\
\hline Transfusional & - & $6(38,0)$ & & $10(40,0)$ & - & \\
\hline Uso de drogas & - & $4(25,0)$ & & $5(24,0)$ & - & \\
\hline Vertical & $3(38,0)$ & - & & - & $1(25,0)$ & \\
\hline Tratamento cirúrgico & $1(12,0)$ & $2(13,0)$ & & $4(16,0)$ & - & \\
\hline Outros & - & $1(6,0)$ & & $2(33,0)$ & - & \\
\hline
\end{tabular}

a:Teste qui-quadrado; b:Teste exato de Fisher; Fonte: Fichas de investigação de hepatites virais, HU-UFPI, 2014-2017. HBsAg: antígeno de superfície do vírus da hepatite B. Indica infecção atual da hepatite B; Anti-HCV: detecta a hepatite C

\section{DISCUSSÃO}

No presente estudo, a análise ratificou, em grande parte, o perfil sociodemográfico e epidemiológico já descrito na literatura nacional e internacional quanto às hepatites $\mathrm{B}$ e C. A distribuição etária se apresentou com predominância na população acima dos 60 anos, com destaque também para aqueles entre 40 a 59 anos. De acordo com análise realizada no Rio Grande do Norte ${ }^{(10)}$ quanto ao vírus da hepatite B (VHB), registrou-se sua associação com o aumento da idade, pois os mecanismos de transmissão envolvem aspectos comportamentais adquiridos ao longo da vida, como a atividade sexual de risco, uso de drogas injetáveis, exposição a sangue e hemoderivados, cuja associação são capazes de gerar efeito cumulativo de riscos para adquirir o VHB, condições também aplicáveis à transmissão do HCV.

Assim como na atual pesquisa, a análise realizada com indivíduos infectados pelo vírus da hepatite $\mathrm{B}^{(4)}$ apontou que $55,1 \%(n=462)$ eram do sexo masculino. Destacou-se, em estudo sobre o perfil sociodemográfico relacionado à hepatite $\mathrm{B}^{(11)}$, que o quantitativo considerável do VHB entre pessoas do sexo masculino ocorre pela maior suscetibilidade a comportamentos de risco, tais como múltiplos parceiros, relação sexual sem proteção e falta da devida atenção à importância da vacinação, fatores que devem ser considerados quando se busca promover ações de saúde que objetivam o controle de doenças transmissíveis como as hepatites virais.

Quanto à raça/cor houve predomínio de pacientes pardos. De acordo com o registro nacional sobre as hepatites virais $^{(6)}$, em 2017, a população parda representou, respectivamente, para hepatites B e C, $41,2 \%$ e $31,8 \%$ dos casos, e identificou-se que as proporções das notificações de casos entre essas pessoas são ascendentes quando comparadas com notificações entre pessoas de raça/cor branca, que é decrescente ao analisar o período de 1999 a 2017.

Em relação à escolaridade, verificou-se no presente estudo que a maioria dos indivíduos possuía o ensino fundamental completo ou menos, o que corrobora o estudo realizado na Bahia, em que a população infectada pelos vírus $\mathrm{B}$ e $\mathrm{C}$ possuía baixa ou nenhuma escolaridade ${ }^{(12)}$.

Também na Bahia ${ }^{(13)}$ foi demonstrado percentual significativo $(91,39 \%)$ de indivíduos com hepatite $C$ que residiam em regiões urbanas, semelhante aos achados deste estudo, em que se acredita que tal condição esteja associada à facilidade de acesso aos serviços de saúde e ao diagnóstico e tratamento das hepatites $\mathrm{B}$ e $\mathrm{C}$.

No tocante à ocupação, a maioria dos casos notificados na presente pesquisa era de aposentados, fato que pode estar relacionado à predominância de pacientes idosos. Estudo sobre o perfil epidemiológico de pacientes portadores do HCV identificou maior percentual em indivíduos aposentados $(37,84 \%)$ e com idade maior que 60 
anos, já que a população pertencente à faixa etária pode ter sido exposta a algum tipo de procedimento cirúrgico com seringas esterilizadas de forma inadequada e transfusão sanguínea feita até o ano de 1993, quando ainda não havia triagem para hepatite $C$ nos bancos de sangue ${ }^{(14)}$.

No Brasil, a vacinação contra a hepatite B foi implementada a partir de 1989, pelo Programa Nacional de Imunizações, proporcionando a consequente redução da endemicidade da doença a partir da década de 1990, sendo a vacina a principal medida de prevenção da infecção pelo VHB. Por ser uma doença imunoprevinível e ter vacina amplamente disponibilizada nos serviços de saúde para todas as faixas etárias, torna-se preocupante, constatar-se, neste estudo, o número de pacientes que não iniciou o esquema de vacinação. Observa-se que o desconhecimento sobre a patologia, a baixa suscetibilidade percebida relacionada à doença e o baixo nível de escolaridade são fatores que ocasionam a baixa adesão à vacina contra hepatite $\mathrm{B}^{(11)}$

Também se pode observar que $4,1 \%$ dos pacientes tiveram outras IST associadas à hepatite B/C. Sabe-se que ser portador de IST é fator de risco para essa coinfecção, visto que esses indivíduos costumam tem relações sexuais desprotegidas, múltiplos parceiros, além de consumir drogas injetáveis, expondo-se mais ao risco de adoecimento pelas infecções sexualmente transmissíveis ${ }^{(15)}$.

Identificou-se no atual estudo que $78,6 \%$ dos participantes foram submetidos a medicamentos injetáveis, porém não foram encontrados estudos que associassem o uso desses medicamentos com as hepatites $B$ e $C$, uma vez que, ao se utilizar tal forma de administração medicamentosa, tem-se o cuidado de manuseá-los em seringas e agulhas descartáveis. Em contrapartida, o compartilhamento de drogas injetáveis é comprovadamente associado à contaminação pelo vírus da hepatite $\mathrm{B}^{(16)}$.

No caso da hepatite $C$, em países desenvolvidos, observa-se que o modo de transmissão se dá, predominantemente, pelo uso de drogas injetáveis, principalmente pelo compartilhamento de agulhas ${ }^{(17)}$. Contudo, nesta pesquisa, somente $10,2 \%$ dos casos declararam o contato com as drogas injetáveis, fato semelhante ao encontrado em estudo realizado no sudoeste baiano ${ }^{(13)}$, em que somente $10,52 \%$ dos entrevistados utilizavam algum tipo de droga.

Estudo realizado em um hospital do Paquistão confirma que tratamentos dentários e cirúrgicos são fatores de risco responsáveis pelo aumento da prevalência de hepatite $\mathrm{B}$ e $\mathrm{C}^{(18)}$. Nesta análise, verificou-se que a exposição a esses fatores foi frequente, porém, para outros autores ${ }^{(11,19)}$, o índice de transmissão por essas condições se reduz quando se utiliza as normas adequadas de biossegurança, que vão desde a segurança do paciente até a do profissional.

Representado por uma grande variedade genética, o HCV possui vários vírus circulantes, organizando-se em sete genótipos e cerca de 67 subtipos virais ${ }^{(20)}$. $O$ exame de genotipagem torna-se relevante para a avaliação clínico-laboratorial na definição da estratégia de tratamento adequado da hepatite $\mathrm{C}$ crônica, sendo recomendado para os casos com indicação de tratamento ${ }^{(21)}$.

Neste estudo, os genótipos relacionados à infecção pela hepatite $C$ apresentam consonância com o perfil existente no Brasil e no mundo, em que o mais prevalente é o genótipo 1, seguido do genótipo $3^{(13,22-25)}$.

Quanto à forma clínica, predominou a hepatite crônica, dado que corrobora estudos realizados em um estado no Nordeste do Brasil, a partir da análise do perfil dos portadores de hepatite B e C, em que os casos crônicos corresponderam a $76,67 \%$ e $99,52 \%$, respectivamente ${ }^{(13,26)}$.

$\mathrm{O}$ risco de cronificação pelo vírus $\mathrm{B}$ depende da idade na qual ocorre a infecção. Em menores de 1 ano chega a $90 \%$, entre 1 e 5 anos o risco está entre 20 e $50 \%$, e na idade adulta é de cerca de $10 \%$. Para o vírus C, a taxa de cronificação fica entre 60 e $90 \%$, sendo maior quando envolve alguns fatores, como sexo masculino, imunodeficiências e idade maior que 40 anos $^{(22)}$.

A grande maioria dos casos associados à hepatite crônica se deve à natureza silenciosa e oligo/assintomática. Outro fator importante para a cronificação é a maior ocorrência da infecção em pessoas empobrecidas e/ou em situação de vulnerabilidade, que, em geral, têm acesso restrito aos serviços públicos de saúde e o diagnóstico da infecção somente é realizado em uma fase avançada da doença ${ }^{(17,22,27)}$.

Quanto à provável fonte ou mecanismo de transmissão dos casos notificados para as hepatites $\mathrm{B}$ e $\mathrm{C}$, dados divulgados em 2018 demonstram que em mais da metade dos casos essa informação foi registrada como "ignorada", dificultando uma melhor avaliação sobre as prováveis fontes de infecção, semelhante aos dados obtidos neste estudo. Acredita-se que este fato possa estar relacionado à dificuldade que se tem de estabelecer um fator único como o responsável pelo desenvolvimento da infecção( ${ }^{(6)}$.

Em outros estudos realizados sobre hepatites $B$ e $C^{(27,28)}$ se sobressaem a transmissão por via sexual e transfusional e o uso de drogas injetáveis, dados que corroboram os resultados desta pesquisa, que apresentou, além desses mecanismos de transmissão, destaque para a via cirúrgica e a transmissão vertical, sendo esta última associada estatisticamente à hepatite $B$. 
Em análise realizada na Argentina ${ }^{(29)}$ observou-se predomínio do comportamento sexual como fator de risco para hepatite $\mathrm{B}$ e, para a hepatite $\mathrm{C}$, fatores associados à dependência de drogas intravenosas, transfusão e cirurgia. Tais achados assemelham-se aos identificados nesta análise, quando, ao verificar associação estatística significativa por vírus, os casos positivos para hepatite B tiveram a via sexual como a principal possível fonte de infecção, enquanto a via transfusional foi a fonte mais frequente para a hepatite $C$.

Por fim, como limitações deste estudo pode-se destacar: o grande número de variáveis ignoradas registradas nas fichas de investigação; o baixo número de associações estatísticas significativas, que podem estar relacionadas ao número restrito de notificações de hepatites $\mathrm{B}$ e $\mathrm{C}$ confirmadas no período analisado; além do uso de dados secundários, que podem sofrer interferências com relação à qualidade das informações. Dessa forma, espera-se que esta pesquisa possa contribuir com ações que favoreçam a promoção em saúde, prevenção e controle das hepatites virais no estado do Piauí, já que a partir do conhecimento do perfil clínico e epidemiológico dos infectados é possível propor medidas eficazes para o combate a esse importante problema de saúde pública.

\section{CONCLUSÃO}

Constatou-se uma maior prevalência das hepatites virais hospitalares em pacientes idosos, do sexo masculino, com baixa escolaridade, que já apresentava a forma crônica da doença, sendo o vírus $C$ o mais frequente.

Destacou-se, ainda, que a maioria dos pacientes não era imunizada contra a hepatite $B$, reforçando a importância da intensificação de campanhas educativas e de sensibilização voltadas para a imunização contra o vírus B. Ademais, foram verificadas associações estatisticamente significativas entre o VHB e HCV com a faixa etária, procedência, zona de residência, prováveis mecanismos de transmissão e forma clínica, sendo a condição de hepatite crônica a mais prevalente entre os dados.

\section{CONFLITOS DE INTERESSE}

Os autores declaram que não há conflitos de interesses no estudo realizado.

\section{CONTRIBUIÇÕES}

Lígia Maria Cabedo Rodrigues, Érida Zoé Lustosa Furtado e Anne Karoline Nunes de Oliveira contribuíram com a elaboração e delineamento do estudo; a aquisição, análise e interpretação dos dados e a redação e/ou revisão do manuscrito; Jaqueline da Cunha Morais e Maria Tainara dos Santos Resende contribuíram com a aquisição, análise e interpretação dos dados e a redação e/ou revisão do manuscrito; Vanessa Rodrigues da Silva contribuíram com a elaboração e delineamento do estudo.

\section{REFERÊNCIAS}

1. Kubota K, Campos MSA, Pereira LRL. Análise da assistência de saúde aos pacientes com hepatite $B$ e $C$ do estado do Amapá. Rev Ciênc Farm Básica Apl [Internet]. 2014 [acesso em 2018 Jan 21];35(4):597-605. Disponível em: http://serv-bib.fcfar.unesp.br/seer/index.php/Cien_Farm/article/viewFile/3199/3199

2. Mol MPG, Greco DB, Cairncross S, Heller L. Hepatitis B and C in household and health services solid waste workers. Cad Saúde Pública [Internet]. 2015 [acesso em 2018 Jan 21];31(Suppl 1):295-300. Disponível em: http://www.scielo.br/pdf/csp/v31s1/0102-311X-csp-31-s1-0295.pdf

3. Ministério da Saúde (BR), Secretaria de Vigilância em Saúde. Hepatites Virais 2017. Boletim Epidemiológico [Internet]. 2017 [acesso em 2018 Jan 21];48(24). Disponível em: http://www.aids.gov.br/pt-br/pub/2017/ boletim-epidemiologico-de-hepatites-virais-2017

4. Costa PRS Jr, Oliveira HS, Silva LEL, Barbosa RFM, Silva RLF. Infecção por hepatites B e C em um município do estado do Pará. Rev Para Med [Internet]. 2013 [acesso em 2018 Jan 21];27(2). Disponível em: http://files.bvs.br/upload/S/0101-5907/2013/v27n2/a3673.pdf

5. Ferreira CT, Silveira TR. Hepatites virais: aspectos da epidemiologia e da prevenção. Rev Bras Epidemiol [Internet]. 2004 [acesso em 2018 Jan 21];7(4):473-87. Disponível em: http://www.scielo.br/pdf/\%0D/rbepid/ v7n4/10.pdf 
6. Ministério da Saúde (BR), Secretaria de Vigilância em Saúde. Hepatites Virais 2018. Bol Epidemiol [Internet]. 2018 [acesso em 2018 Ago 08];49(31). Disponível em: http://portalarquivos2.saude.gov.br/images/pdf/2018/ julho/05/Boletim-Hepatites-2018.pdf

7. Ministério da Saúde (BR). Portaria $n^{\circ} 2446$, de 11 de novembro de 2014. Redefine a Política Nacional de Promoção da Saúde (PNPS) [Internet]. 2014 [acesso em 2018 Ago 08]. Disponível em: http://bvsms.saude. gov.br/bvs/saudelegis/gm/2014/prt2446_11_11_2014.html

8. Silva ACLG, Tozatti F, Welter AC, Miranda CDC. Incidência e mortalidade por hepatite B, de 2001 a 2009: uma comparação entre o Brasil, Santa Catarina e Florianópolis. Cad Saúde Colet [Internet]. 2013 [acesso em 2018 Jan 21];21(1):34-9. Disponível em: http://www.scielo.br/pdf/cadsc/v21n1/a06.pdf

9. World Health Organization. Guidelines for the screening care and treatment of persons with chronic hepatitis $\mathrm{c}$ infection: updated version guidelines for the screening care and treatment of persons with chronic hepatitis C infection [Internet]. Geneva: World Health Organization; 2016 [acesso em 2018 Ago 08]. Disponível: http:// apps.who.int/iris/bitstream/handle/10665/205035/9789241549615_eng.pdf;jsessionid=D8F3B42150DD42EEA A9D303A1BAE79EC? sequence $=1$

10. Justino EMG, Bacelar SSS, Araújo SD, Oliveira RM, Almeida EB, Sousa GA, et al. Perfil de portadores de hepatite B em um serviço de referência: estudo retrospectivo. Rev Bras Promoç Saúde [Internet]. 2014 [acesso em 2018 Abr 03];27(1):53-61. doi: 10.5020/18061230.2014.p53

11. Gusmão BM, Pereira FS, Rocha AP, Fernandes MBS, Dias OV, Costa SM. Análise do perfil sociodemográfico de notificados para hepatite B e imunização contra a doença. Rev Pesqui (Univ Fed Estado Rio J) [Internet]. 2017 [acesso em 2018 Abr 13];9(3):627-33. doi: 10.9789/2175-5361.2017.v9i3.627-633

12. Ferreira CO, Davoglio RS, Silva AA, Viana ASA, Rezende REAR, Gomes AVTM. Perfil epidemiológico dos usuários de um centro de testagem e aconselhamento da Bahia. Rev Baiana Saúde Pública [Internet]. 2016 [acesso em 2018 Abr 13];40(2):388-409. doi: 10.22278/2318-2660.2016.v40.n2.a1980

13. Morais MTM, Oliveira TJ. Perfil epidemiológico e sociodemográfico de portadores de Hepatite $C$ de um município do Sudoeste Baiano. Revista Saúde.Com [Internet]. 2015 [acesso em 2018 Abr 20];11(2):137-46. Disponível em: www.uesb.br/revista/rsc/v11/v11n2a04.pdf

14. Mello JC, Motta TP, Santos MC. Perfil epidemiológico de portadores de Hepatite $C$ do núcleo hospitalar epidemiológico do sul de Brasil. Rev Ensaios Ciênc [Internet]. 2011 [acesso em 2018 Abr 20];15(3):55-64. Disponível em: http://www.redalyc.org/pdf/260/26021120006.pdf

15. Santos OP, Souza MR, Borges CJ, Noll M, Lima FC, Barros PS. Hepatites B, C e sífilis: prevalência e características associadas à coinfecção entre soropositivos. Cogitare Enferm [Internet]. 2017 [acesso em 2018 Abr 22];22(3):1-8. doi: 10.5380/ce.v22i3.51693

16. Teles SA. Viral hepatitis: a challenge for nursing. Rev Bras Enferm [Internet]. 2017 [acesso em $2018 \mathrm{Abr}$ 22];70(2):231-2. doi: 10.1590/0034-7167.2017700201

17. Sá LC, Araújo TME, Griep RH, Campelo V, Monteiro CFS. Soroprevalência da Hepatite C e fatores associados em usuários de crack. Rev Latinoam Enferm [Internet]. 2013 [acesso em 2018 Abr 22];21(6):1195-202. doi: 10.1590/0104-1169.3126.2354

18. Shafiq M, Nadeem M, Sattar Z, Khan SM, Faheem SM, Ahsan I, et al. Identification of risk factors for hepatitis B and C in Peshawar, Pakistan. HIV AIDS [Internet]. 2015 [acesso em 2018 Abr 22];7:223-31. doi: 10.2147I HIV.S67429

19. Pereira FS, Gusmão BM, Rocha AP, Fernandes MBS, Dias OV, Costa SM. Estilo de vida e exposição à material biológico entre notificados com hepatite B. J Health Biol Sci [Internet]. 2016 [acesso em 2018 Abr 15];4(2):117-22. doi: 10.12662/2317-3076jhbs.v4i2.714.p117-122.2016

20. Smith DB, Bukh J, Kuiken C, Muerhoff AS, Rice CM, Stapleton JT, et al. Expanded classification of hepatitis C virus into 7 genotypes and 67 subtypes: update criteria and genotype assignment web resource. Hepatology [Internet]. 2014 [acesso em 2018 Ago 08];59:318-27. Disponível em: https://www.ncbi.nlm.nih.gov/pmc/ articles/PMC4063340/

21. Ministério da Saúde (BR), Secretária de Vigilância em Saúde, Departamento de Vigilância, Prevenção e 
Controle das Infecções Sexualmente Transmissíveis, do HIV/Aids e das Hepatites Virais. Protocolo Clínico e Diretrizes Terapêuticas para hepatite $C$ e coinfecções [Internet]. Brasília: Ministério da Saúde; 2017 [acesso em 2018 Ago 08]. Disponível em: http://www.aids.gov.br/pt-br/pub/2017/protocolo-clinico-e-diretrizesterapeuticas-para-hepatite-c-e-coinfeccoes

22. Ministério da Saúde (BR), Secretaria de Vigilância em Saúde, Coordenação-Geral de Desenvolvimento da Epidemiologia em Serviços. Guia de vigilância em saúde [Internet]. Brasília: Ministério da Saúde; 2017 [acesso em 2018 Ago 08]. Disponível em: http://bvsms.saude.gov.br/bvs/publicacoes/guia_vigilancia_saude_ volume_2.pdf

23. Coutinho BC, Soprani M, Pandolfi NC, Tonini RC. Prevalência de genótipos em portadores crônicos do vírus da hepatite C. Rev Soc Bras Clin Med. 2017;15(1):11-4.

24. Malacrida AM, Miyamoto SK, Menezes HC, Santos MF Neta, Bertolini DA. Prevalência dos genótipos do vírus da Hepatite $C$ da macrorregião Noroeste do Estado do Paraná, Brasil. Saúde e Pesqui [Internet]. 2016 [acesso em 2018 Abr 25];9(2):381-8. doi: 10.17765/1983-1870.2016v9n2p381-388

25. Messina JP, Humphreys I, Flaxman A, Brown A, Cooke GS, Pybus OG, et al. Global distribution and prevalence of hepatitis C virus genotypes. Hepatology [Internet]. 2015 [acesso em 2018 Abr 25];61(1):77-87. doi: 10.1002/hep.27259

26. Santos O, Gómez A, Vizcaíno V, Casas MC, Ramírez MP, Olaya P. Genotipos circulantes del virus de la hepatitis C en Colombia. Biomédica [Internet]. 2017 [acesso em 2018 Ago 08];37(1):22-7. doi: 10.7705/ biomedica.v37i1.3173

27. Santos ACS, Morais MTM. Perfil epidemiológico e sociodemográfico dos portadores de hepatite $B$ de um município do Sudoeste Baiano. Revista Saúde.Com [Internet]. 2018 [acesso em 2018 Ago 08];14(1):1073-80. doi: $10.22481 /$ rsc.v14i1.537

28. Alves MR, Silva DM, Souza TO, Oliveira YNS, Nery AA, Casotti CA. Perfil epidemiológico dos casos de hepatite $C$ em uma diretoria regional de saúde da Bahia. Rev Pesqui (Univ Fed Estado Rio J) [Internet]. 2013 [acesso em 2018 Ago 08];6(3):889-96. Disponível em: www.redalyc.org/pdf/5057/505750623004.pdf

29. Vladimirsky SN. Vigilancia epidemiológica de las hepatitis virales en la Argentina. Salud(i)ciência [Internet]. 2015 [acesso em 2018 Ago 08];21(3):314-6. Disponível em: www.siicsalud.com/dato/sic/213/136486.pdf

\section{Endereço para correspondência:}

Lígia Maria Cabedo Rodrigues

Hospital Universitário da Universidade Federal do Piauí - HU-UFPI

Campus I - Campus Universitário Ministro Petrônio Portela, SG 07 s/n

Bairro: Ininga

CEP: 64049-550 - Teresina - PI - Brasil

E-mail: ligiacabedo@yahoo.com.br

Como citar: Rodrigues LMC, Furtado EZL, Oliveira AKN, Morais JC, Resende MTS, Silva VR. Mapeamento epidemiológico das hepatites hospitalares. Rev Bras Promoç Saúde. 2019;32:8714. 